\title{
ON THE DEFINITION OF SIGNIFICANT MULTIPLIC- ITY FOR CONTINUOUS TRANSFORMATIONS
}

\author{
BY \\ EARL J. MICKLE \\ INTRODUCTION
}

Let $T$ be a continuous transformation from the unit square $Q: 0 \leqq u \leqq 1$, $0 \leqq v \leqq 1$, in the $u v$-plane into Euclidean 3 -space $S_{3}$ and let $x$ designate a point in $S_{3}$. If $T^{-1}(x) \neq \varnothing$ then each component of $T^{-1}(x)$ is called a maximal model continuum under $T$. The reader is referred to Rado [6] for background material and definitions (square brackets refer to the bibliography at the end of this paper). For a set $E \subset Q$ let $N^{*}(x, T, E)$ denote the number (possibly $+\infty$ ) of maximal model continua under $T$ that intersect $T^{-1}(x) \cap E$. Let $A(T)$ denote the Lebesgue area of the surface represented by $T$. If $T$ is a plane transformation, i.e., $T(Q)$ lies in a plane, and $\varepsilon^{*}$ is the union of the essential maximal model continua under $T$, then (see Cesari [1] and Rado [7])

$$
A(T)=\int N^{*}\left(x, T, \mathcal{E}^{*}\right) d L_{2}
$$

where the integration is taken with respect to Lebesgue planar measure $L_{2}$ in the plane containing $T(Q)$.

The reader is referred to Federer [4] for a discussion and a solution of the problem of introducing a suitable multiplicity function and a suitable measure in $S_{3}$ such that a relationship as given above for plane transformations holds for a continuous transformation from $Q$ into $S_{3}$. By using the concept of an essential maximal model continuum for plane transformations the writer [5] defined an essential maximal model continuum under a continuous transformation $T$ from $Q$ into $S_{3}$. If $\mathcal{E}$ is the union of these essential maximal model continua under $T$ then

$$
A(T)=\int N^{*}(x, T, \varepsilon) d H^{2}
$$

where the integration is taken with respect to Hausdorff 2-dimensional measure $H^{2}$ in $S_{3}$. Rado [9] showed that a set $\mathcal{E}$ of essential maximal model continua could be defined in a simpler manner than that used by the writer in [5] such that (1) holds for this set $\varepsilon$. However, both the definition of Rado and the definition of the writer depend on the coordinate system.

It is the purpose of this paper to use the concept of an essential maximal

Received by the editors January 30, 1956. 
model continuum under a plane transformation to define a significant maximal model continuum under a continuous transformation $T$ from $Q$ into $S_{3}$ such that the following two conditions are satisfied. (i) The definition of a significant maximal model continuum under $T$ is independent of the coordinate system. (ii) If $S$ is the union of the significant maximal model continua under $T$ then

$$
A(T)=\int N^{*}(x, T, \delta) d H^{2}
$$

where the integration is taken with respect to Hausdorff 2-dimensional measure $H^{2}$ in $S_{3}$. We use the term significant instead of essential because in the case of plane transformations the two concepts do not agree.

\section{Significant maximal model continua}

1.1. As mentioned before we shall denote Euclidean $x_{1} x_{2} x_{3}$-space by $S_{3}$ and points in $S_{3}$ by $x$. Points on the unit sphere $U: x_{1}^{2}+x_{2}^{2}+x_{3}^{2}=1$ will be denoted by $P . S_{2}(P)$ is the plane through the origin which is perpendicular to the line joining $P$ to the origin. Points in $S_{2}(P)$ will be denoted by $x_{P}$. $\pi_{P}: S_{3} \Rightarrow S_{2}(P)$ is the orthogonal projection from $S_{3}$ onto $S_{2}(P)$. The open sphere with center at $x$ and radius $r>0$ will be denoted by $s(x, r)$.

1.2. We denote by $H^{2}(E)$ the Hausdorff 2-dimensional measure of a set $E \subset S_{3}$. We denote by $\Gamma$ the class of $H^{2}$-measurable sets. $\Gamma$ contains all open, Borel and analytic sets. For a set $E$ lying in a plane we denote by $L_{2}(E)$ the Lebesgue exterior planar measure of $E$. For $P \in U$ we define

$$
\Gamma_{P}=\left[E \mid E \in \Gamma, L_{2} \pi_{P}(E)=0\right] \text {. }
$$

For a set $E \in \Gamma$ we define

$$
H_{P}(E)=\text { gr.l.b. } H^{2}\left(E-E_{P}\right) \text { for sets } E_{P} \in \Gamma_{P} \text {. }
$$

Then (see Mickle [5]) $H_{P}(E)$ is a measure for sets $E \in \Gamma$ and for a set $E \in \Gamma$ the following conditions are satisfied. (i) $H_{P}(E) \leqq H^{2}(E)$. (ii) $H_{P}(E) \geqq L_{2} \pi_{P}(E)$. (iii) There exist sets $E^{\prime}, E^{\prime \prime} \in \Gamma$ such that $E=E^{\prime} \cup E^{\prime \prime}, E^{\prime} \cap E^{\prime \prime}=\varnothing, H_{P}(E)$ $=H_{P}\left(E^{\prime}\right)=H^{2}\left(E^{\prime}\right)$ and $H_{P}\left(E^{\prime \prime}\right)=0$. (iv) If $L_{2} \pi_{P}(E)=0$ then $H_{P}(E)=0$.

1.3. For $E \in \Gamma, x \in S_{3}, P \in U$ we define

$$
\begin{array}{rlrl}
h(x, E) & =\underset{r \rightarrow 0^{+}}{\limsup } H^{2}[E \cap s(x, r)] / \pi r^{2}, h_{P}(x, E) & =\underset{r \rightarrow 0^{+}}{\limsup } H_{P}[E \cap s(x, r)] / \pi r^{2}, \\
D(E) & =[x \mid h(x, E)>0], & D_{P}(E) & =\left[x \mid h_{P}(x, E)>0\right] .
\end{array}
$$

1.4. Lemma. Let $\Phi(E)$ be a measure for sets $E \in \Gamma$. For each pair of positive integers $n$ and $m$ the set

$$
G_{n, m}(E, \Phi)=\left\{x \mid \Phi[E \cap s(x, r)]>\pi r^{2} / n \text { for some } r, 0<r<1 / m\right\}
$$

is an open set. 
Proof. If $x^{*} \in G_{n, m}(E, \Phi)$ then there are numbers $r^{*}, r, 0<r^{*}<r<1 / m$ such that $\Phi\left[E \cap s\left(x^{*}, r^{*}\right)\right]>\pi r^{2} / n$. Hence there is a $\rho>0$ such that for $x \in s\left(x^{*}, \rho\right)$ we have $s(x, r) \supset s\left(x^{*}, r^{*}\right)$. Thus $\Phi[E \cap s(x, r)] \geqq \Phi\left[E \cap s\left(x^{*}, r^{*}\right)\right]$ $>\pi r^{2} / n, s\left(x^{*}, \rho\right) \subset G_{n, m}(E, \Phi)$ and $G_{n, m}(E, \Phi)$ is an open set.

1.5. LEmma. For $E \in \Gamma, P \in U$ we have the following conditions satisfied by the sets $D(E)$ and $D_{P}(E)$ defined in $\S 1.3$. (i) $D(E)$ and $D_{P}(E)$ are Borel sets. (ii) $H^{2}[E-D(E)]=0$. (iii) $H_{P}\left[E-D_{P}(E)\right]=0$. (iv) If $H^{2}(E)<\infty$ then $H^{2}[D(E)-E]=0$.

Proof. (i) follows from the lemma in $\$ 1.4$ since

$$
D(E)=\bigcup_{n=1}^{\infty} \bigcap_{m=1}^{\infty} G_{n, m}\left(E, H^{2}\right), \quad D_{P}(E)=\bigcup_{n=1}^{\infty} \bigcap_{m=1}^{\infty} G_{n, m}\left(E, H_{P}\right) .
$$

(ii) and (iv) are well known but the proofs of (ii), (iii), and (iv) can be found in Mickle [5].

1.6. Let $Q$ be the unit square $0 \leqq u \leqq 1,0 \leqq v \leqq 1$ in the $u v$-plane and let $T$ be a continuous transformation from $Q$ into $S_{3}$. For a set $E \subset Q, N(x, T, E)$ will denote the number (possibly $+\infty$ ) of points in $T^{-1}(x) \cap E$. As mentioned in the introduction a component of $T^{-1}(x) \neq \varnothing$ is called a maximal model continuum (m.m.c.) under $T$. For a set $E \subset Q, N^{*}(x, T, E)$ will denote the number (possibly $+\infty$ ) of m.m.c.'s under $T$ that intersect $T^{-1}(x) \cap E$. If $E$ is an analytic subset of $Q$ then by well known theorems in measure theory $N(x, T, E)$ and $N^{*}(x, T, E)$ are $H^{2}$ and $H_{P}$ measurable functions.

1.7. For a continuous transformation $T$ from $Q$ into $S_{3}$ and for $P \in U$ we set $\mathcal{E}_{P}(T)$ to be the union of all the essential maximal model continua (see Rado [6, pp. 281-282]) under the continuous plane transformation $T_{P}=\pi_{P} T$ from $Q$ into the plane $S_{2}(P) . \varepsilon_{P}(T)$ is a Borel set (see Rado [6, p. 296]).

1.8. For $E \in \Gamma, P \in U$ and pair of positive integers $n$ and $m$ we set

$$
G_{n, m}(E, P)=\left\{x \mid H_{P}[E \cap s(x, r)]>\pi r^{2} / n \text { for some } r, 0<r<1 / m\right\} .
$$

For a continuous transformation $T$ from $Q$ into $S_{3}$, a Borel set $B$ in the $u v$-plane and $\mathcal{E}_{P}=\mathcal{E}_{P}(T)$ we set

$$
D^{*}(T, B)=\bigcup_{n=1}^{\infty} \bigcap_{m=1}^{\infty} \bigcup_{P \in U} G_{n, m}\left[T\left(B \cap \varepsilon_{P}\right), P\right] .
$$

1.9. Lemma. For a continuous transformation $T$ from $Q$ into $S_{3}$ and a Borel set $B$ in the uv-plane the set $D^{*}(T, B)$ defined in $\$ 1.8$ satisfies the following conditions. (i) $D^{*}(T, B)$ is a Borel set. (ii) For $P \in U, D_{P}\left[T\left(B \cap \varepsilon_{P}\right)\right] \subset D^{*}(T, B)$.

Proof. (i) follows from the lemma in $\S 1.4$. If $x \in D_{P}\left[T\left(B \cap \varepsilon_{P}\right)\right]$ then there is an $n_{0}$ such that $x \in G_{n_{0}, m}\left[T\left(B \cap \mathcal{E}_{P}\right), P\right]$ for every positive integer $m$. Hence $x \in D^{*}(T, B)$ and (ii) follows.

1.10. Let $\Omega$ be the class of open sets in the $u v$-plane. For a continuous 
transformation $T$ from $Q$ into $S_{3}$ an m.m.c. $\gamma$ under $T$ will be called a significant maximal model continuum (s.m.m.c.) under $T$ if for every open set $O \in \Omega$ such that $\gamma \subset O$ we have that $T(\gamma) \in D^{*}(T, O)$ (see $\$ 1.8$ ). We denote by $\delta(T)$ the union of the s.m.m.c.'s under $T$. Obviously, $s(T)$ is independent of the coordinate system.

1.11. Let $T=l m, m: Q \Rightarrow M, l: M \rightarrow S_{3}$ be a monotone-light factorization of a continuous transformation $T$ from $Q$ into $S_{3}$ (See Rado [6, p. 54]). Let $a_{1}, a_{2}, \cdots$, be a dense denumerable set of points in $M$ and for each pair of positive integers $i$ and $j$ let $s\left(a_{i}, 1 / j\right)$ be the open sphere with center at $a_{i}$ and radius $1 / j$. We set

$$
\Omega_{T}=\left\{O_{i, j} \mid O_{i, j}=m^{-1}\left[s\left(a_{i}, 1 / j\right)\right], i, j=1,2, \cdots,\right\} .
$$

Note that for each $O \in \Omega_{T}, O$ is open relative to $Q$, i.e., there is an $O^{*} \in \Omega$ such that $O=Q \cap O^{*}$.

1.12. Lemma. Let $T$ be an continuous transformation from $Q$ into $S_{3}$. $A n$ m.m.c. $\gamma$ under $T$ is an s.m.m.c. under $T$ if and only if for each $O \in \Omega_{T}$ such that $\gamma \subset O$ we have $T(\gamma) \in D^{*}(T, O)$.

Proof. Let $\gamma$ be an s.m.m.c. under $T$ and let $O \in \Omega_{T}$ be such that $\gamma \subset O$. Then there is an $O^{*} \in \Omega$ such that $O=Q \cap O^{*}$. For $P \in U$, we have $\varepsilon_{P} \subset Q$ and hence $G_{n, m}\left[T\left(O^{*} \cap \mathcal{E}_{P}\right), P\right]=G_{n, m}\left[T\left(O^{*} \cap Q \cap \mathcal{E}_{P}\right), P\right]=G_{n, m}\left[T\left(O \cap \mathcal{E}_{P}\right), P\right]$. Hence $T(\gamma) \in D^{*}\left(T, O^{*}\right)=D^{*}(T, O)$.

Let $\gamma$ be an m.m.c. under $T$ such that for $O \in \Omega_{T}, \gamma \subset O$, we have $T(\gamma)$ $\in D^{*}(T, O)$. Let $O^{*} \in \Omega$ be such that $\gamma \subset O^{*}$. Let $O_{0}^{*}$ be the union of all the m.m.c.'s under $T$ that lie entirely in $O^{*}$. Then $O_{0}^{*}$ is open relative to $Q$. For the monotone transformation $m$ given in $\$ 1.11, m\left(O_{0}^{*}\right)$ is an open set in $M$, $m^{-1} m\left(O_{0}^{*}\right)=O_{0}^{*}$ and $m(\gamma) \in m\left(O_{0}^{*}\right)$. Thus there are integers $i$ and $j$ such that $m(\gamma) \in s\left(a_{i}, 1 / j\right) \subset m\left(O_{0}^{*}\right)$. Thus $\gamma \subset O_{i, j} \subset O_{0}^{*} \subset O^{*}$ and $T(\gamma) \in D^{*}\left(T, O_{i, j}\right)$ $\subset D^{*}\left(T, O^{*}\right)$. Thus $\gamma$ is an s.m.m.c. under $T$.

1.13. Lemma. For a continuous transformation $T$ from $Q$ into $S_{3}, S(T)$ is a Borel set.

Proof. We shall show that for the sets $O_{i, j}, i, j=1,2, \ldots$, defined in $\S 1.11$

$$
S(T)=\bigcap_{j=1}^{\infty} \bigcup_{i=1}^{\infty}\left\{O_{i, j} \cap T^{-1}\left[D^{*}\left(T, O_{i, j}\right)\right]\right\} .
$$

By (i) of the lemma in $\S 1.9$ the right side of (1) is a Borel set. Note that the left side of (1) and the right side of (1) are the union of m.m.c.'s under $T$.

(i) Let $\gamma$ be an s.m.m.c. under $T$. Then for each positive integer $j$ there is a positive integer $i$ such that $\gamma \subset O_{i, j}$ and hence, by the lemma in $\$ 1.12$, such that $T(\gamma) \in D^{*}\left(T, O_{i, j}\right)$. Thus $\gamma$ is in the right side of (1). Hence the left side of (1) is a subset of the right side of (1). 
(ii) Let $\gamma$ be an m.m.c. under $T$ lying in the right side of (1). Let $O \in \Omega_{T}$ be such that $\gamma \subset O$. Then there exists an integer $j_{0}$ such that if $m(\gamma) \in s\left(a_{i}, 1 / j_{0}\right)$ then $s\left(a_{i}, 1 / j_{0}\right) \subset m(O)$. Now there is an integer $i_{0}$ such that

$$
\gamma \subset O_{i_{0}, j_{0}} \cap T^{-1}\left[D^{*}\left(T, O_{i_{0}, j_{0}}\right)\right], \quad T(\gamma) \in D^{*}\left[\left(T, O_{i_{0}, j_{0}}\right)\right],
$$

and hence, since, $O_{i_{0}, j_{0}} \subset O$,

$$
T(\gamma) \in D^{*}\left(T, O_{i_{0}, j_{0}}\right) \subset D^{*}(T, O) .
$$

By the lemma in $\$ 1.12, \gamma$ is an s.m.m.c. under $T$ and the right side of (1) is a subset of the left side of (1).

(1) follows from (i) and (ii).

1.14. Lemma. For a continuous transformation $T$ from $Q$ into $S_{3}$ and $P \in U$,

$$
N^{*}[x, T, s(T)] \geqq N^{*}\left[x, T, \mathcal{E}_{P}(T)\right]
$$

except on a set of $H_{P}$ measure zero.

Proof. The fact that (1) holds except on set of $H_{P}$ measure zero will follow when we have shown that for $\varepsilon_{P}=\varepsilon_{P}(T), \delta=\delta(T)$,

$$
H_{P}\left[T\left(\varepsilon_{P}-\delta\right)\right]=0 .
$$

Let $\gamma$ be an m.m.c. under $T$ such that $\gamma \subset \mathcal{E}_{P}-\delta$. By the lemma in $\S 1.12$ there is a set $O \in \Omega_{T}$ such that $\gamma \subset O, T(\gamma) \in T\left(O \cap \mathcal{E}_{P}\right), T(\gamma) \notin D^{*}(T, O)$. By (ii) of the lemma in $\S 1.9, T(\gamma) \notin D_{P} T\left(O \cap \varepsilon_{P}\right)$. Thus

$$
T\left(\varepsilon_{P}-s\right) \subset \bigcup_{o \in \Omega T}\left\{T\left(O \cap \mathcal{E}_{P}\right)-D_{P}\left[T\left(O \cap \mathcal{E}_{P}\right)\right]\right\} .
$$

By (iii) of the lemma in $\$ 1.5$ and the definition of $\Omega_{T}$ the right side of (3) is the union of a denumerable number of sets of $H_{P}$ measure zero. Thus (2) holds.

1.15. THEOREM. If $T$ is a continuous transformation from $Q$ into $S_{3}$ such that $A(T)=\infty$ then

$$
A(T)=\int N^{*}[x, T, s(T)] d H^{2} .
$$

Proof. $A(T)=\infty$ implies that there is a $P \in U$ such that $A\left(T_{P}\right)=\infty$ (see Cesari [1]). By (i) of $\S 1.2$, the lemma in $\S 1.14$ and (ii) of $\S 1.2$, for $\delta=\delta(T)$, $\varepsilon_{P}=\varepsilon_{P}(T)$ we have

$$
\begin{aligned}
\int N^{*}(x, T, \S) d H^{2} & \geqq \int N^{*}(x, T, \S) d H_{P} \geqq \int N^{*}\left(x, T, \varepsilon_{P}\right) d H_{P} \\
& \geqq \int N^{*}\left(x_{P}, T_{P}, \varepsilon_{P}\right) d L_{2}=A\left(T_{P}\right)=\infty
\end{aligned}
$$




\section{Differentiable essentially absolutely CONTINUOUS TRANSFORMATIONS}

2.1. Let

$$
T: x_{1}=x_{1}(u, v), \quad x_{2}=x_{2}(u, v), \quad x_{3}=x_{3}(u, v), \quad(u, v) \in Q,
$$

be a continuous transformation from $Q$ into $S_{3}$. At a point where the partial derivatives that are involved exist we set

$$
\begin{array}{ll}
J_{1}(u, v)=x_{2 u} x_{3 v}-x_{2 v} x_{3 u}, & J_{2}(u, v)=x_{3 u} x_{1 v}-x_{3 c} x_{1 u}, \\
J_{3}(u, v)=x_{1 u} x_{2 v}-x_{1 v} x_{2 u}, & W(u, v)=\left[J_{1}^{2}+J_{2}^{2}+J_{3}^{2}\right]^{1 / 2} .
\end{array}
$$

If $T$ is such that $W(u, v)$ exists almost everywhere on $Q$, is summable on $Q$ and

$$
A(T)=\iint_{Q} W(u, v) d u d v,
$$

then $T$ is essentially absolutely continuous (see Rado [6, pp. 504-505]). We shall call such a transformation a differentiable absolutely continuous transformation. Throughout this section we shall assume that $T$ is a fixed differentiable essentially absolutely continuous transformation. $B_{0}$ will be a fixed Borel set in $Q^{0}$ (the interior of $Q$ ) such that $W(u, v)$ exists everywhere on $B_{0}$ and $L_{2}\left(Q-B_{0}\right)=0$. For any Borel set $B \subset B_{0}$ we have that (see Federer [3])

$$
\iint_{B} W(u, v) d u d v=\int N(x, T, B) d H^{2} .
$$

For $P \in U, J_{P}(u, v)$ will designate the ordinary Jacobian of the plane mapping $T_{P}$. Then $J_{P}(u, v)$ exists everywhere on $B_{0}$,

$$
A\left(T_{P}\right)=\iint_{Q}\left|J_{P}(u, v)\right| d u d v,
$$

and for any Borel set $B C B_{0}$

$$
\iint_{B}\left|J_{P}(u, v)\right| d u d v=\int N\left(x_{P}, T_{P}, B\right) d L_{2} .
$$

2.2. Lemma. For any Borel set $B \subset Q$ and $P \in U$,

$$
\iint_{B}\left|J_{P}(u, v)\right| d u d v=\int N\left[x_{P}, T_{P}, B \cap \varepsilon_{P}(T)\right] d L_{2} .
$$

Proof. Set $B^{*}=Q-B, \varepsilon_{P}=\varepsilon_{P}(T)$. Since $L_{2}\left(\varepsilon_{P}-B_{0}\right)=0$ we have that $L_{2}\left[T_{P}\left(\varepsilon_{P}-B_{0}\right)\right]=0$ (see Rado [8]). Thus

$$
A\left(T_{P}\right)=\int N\left(x_{P}, T_{P}, \varepsilon_{P}\right) d L_{2}=\int N\left(x_{P}, T_{P}, B_{0} \cap \varepsilon_{P}\right) d L_{2} .
$$


From (2) we thus have

(3) $A\left(T_{P}\right)=\int N\left(x_{P}, T_{P}, B_{0} \cap B \cap \varepsilon_{P}\right) d L_{2}+\int N\left(x_{P}, T_{P}, B_{0} \cap B^{*} \cap \varepsilon_{P}\right) d L_{2}$.

From (3) and (4) of $\$ 2.1$

$$
A\left(T_{P}\right)=\int N\left(x_{P}, T_{P}, B_{0} \cap B\right) d L_{2}+\int N\left(x_{P}, T_{P}, B_{0} \cap B^{*}\right) d L_{2} .
$$

Subtracting (3) from (4) gives

$$
\begin{aligned}
& {\left[\int N\left(x_{P}, T_{P}, B_{0} \cap B\right) d L_{2}-\int N\left(x_{P}, T_{P}, B_{0} \cap B \cap \mathcal{E}_{P}\right) d L_{2}\right]} \\
& +\left[\int N\left(x_{P}, T_{P}, B_{0} \cap B^{*}\right) d L_{2}-\int N\left(x_{P}, T_{P}, B_{0} \cap B^{*} \cap \mathcal{E}_{P}\right) d L_{2}\right]=0 .
\end{aligned}
$$

Since each of the quantities in the square brackets in (5) is non-negative,

$$
\int N\left(x_{P}, T_{P}, B_{0} \cap B\right) d L_{2}=\int N\left(x_{P}, T_{P}, B_{0} \cap B \cap \varepsilon_{P}\right) d L_{2} .
$$

Since $L_{2} T_{P}\left(\varepsilon_{P}-B_{0}\right)=0$ the right side of (6) is equal to the right side of (1). Since $L_{2}\left(B-B_{0}\right)=0$, by (4) of $\$ 2.1$, the left side of (6) is equal to the left side of (1). (1) thus follows from (6).

2.3. Lemma. Let $B_{0}^{*}$ be the union of all the m.m.c.'s under $T$ that intersect $B_{0}$. Then

$$
A(T)=\int N^{*}\left(x, T, B_{0}^{*}\right) d H^{2} .
$$

Proof. Since $B_{0}^{*}$ is an analytic set the integral on the right side of (1) exists either finite or infinite. Let $F$ be the union of all the nondegenerate m.m.c.'s under $T$. Then (1) will follow from (1) and (2) of $\$ 2.1$ if we establish the relationship

$$
H^{2}\left[T\left(F \cap B_{0}\right)\right]=0 .
$$

$F$ is a Borel set. Since $F$ is the union of nondegenerate m.m.c.'s under $T$, for $P \in U, \varepsilon_{P}=\varepsilon_{P}(T)$, we have that $N\left(x_{P}, T_{P}, F \cap \varepsilon_{P}\right)$ is either 0 or $+\infty$ for each point $x_{P} \in S_{2}(P)$. Thus, since $\left|J_{P}(u, v)\right|$ is summable, by the lemma in $\S 2.2$,

$$
\int N\left(x_{P}, T_{P}, F \cap \varepsilon_{P}\right) d L_{2}=0 \text { for } \quad P \in U
$$

Let $U^{*}$ be the set of points $(1,0,0),(0,1,0),(0,0,1)$ on $U$. By $(2)$ of $\S 2.1$ and the lemma in $\$ 2.2$ 


$$
\begin{aligned}
H^{2}\left[T\left(F \cap B_{0}\right)\right] & \leqq \int N\left(x, T, F \cap B_{0}\right) d H^{2}=\iint_{F \cap_{B_{0}}} W(u, v) d u d v \\
& =\iint_{F} W(u, v) d u d v \leqq \sum_{P \in U^{*}} \iint_{F}\left|J_{P}(u, v)\right| d u d v \\
& =\sum_{P \in U^{*}} \int N\left(x_{P}, T_{P}, F \cap \varepsilon_{P}\right) d L_{2} .
\end{aligned}
$$

(2) thus follows from (3) and (4) and (1) holds.

2.4. Lemma. For $P \in U, E \in \Gamma$ and any Borel set $B$ in the uv-plane

$$
H_{P}\left[T\left(B \cap \varepsilon_{P}\right) \cap E\right]=H_{P}\left[T\left(B \cap B_{0}\right) \cap E\right] .
$$

Proof. Since $L_{2}\left(B-B_{0}\right)=0$ we have (see Rado [8]) $L_{2} \pi_{P} T\left[\left(B-B_{0}\right) \cap E_{P}\right]$ $=L_{2} T_{P}\left[\left(B-B_{0}\right) \cap \mathcal{E}_{P}\right]=0$. By (iv) of $\$ 1.2$

$$
H_{P}\left\{T\left[\left(B-B_{0}\right) \cap \varepsilon_{P}\right]\right\}=0 .
$$

Thus

$$
\begin{aligned}
H_{P}\left[T\left(B \cap \mathcal{E}_{P}\right)\right. & \cap E] \leqq H_{P}\left[T\left\{\left[\left(\mathrm{~B}-B_{0}\right) \cap \mathcal{E}_{P}\right] \cup\left(B \cap B_{0}\right)\right\} \cap E\right] \\
& \leqq H_{P}\left\{T\left[\left(B-B_{0}\right) \cap \varepsilon_{P}\right] \cap E\right\}+H_{P}\left[T\left(B \cap B_{0}\right) \cap E\right] \\
& =H_{P}\left[T\left(B \cap B_{0}\right) \cap E\right] .
\end{aligned}
$$

For $B^{*}=\left(B-\varepsilon_{P}\right) \cap B_{0}, N\left(x_{P}, T_{P}, B^{*} \cap \varepsilon_{P}\right)=0$. Hence, by (4) of $\S 2.1$ and the lemma in $\$ 2.2$,

$$
\begin{aligned}
L_{2} \pi_{P} T\left(B^{*}\right) & =L_{2} T_{P}\left(B^{*}\right) \leqq \int N\left(x_{P}, T_{P}, B^{*}\right) d L_{2}=\iint_{B *}\left|J_{P}(u, v)\right| d u d v \\
& =\int N\left(x_{P}, T_{P}, B^{*} \cap \varepsilon_{P}\right) d L_{2}=0 .
\end{aligned}
$$

By (iv) of $\S 1.2$

$$
H_{P}\left\{T\left[\left(B-\varepsilon_{P}\right) \cap B_{0}\right]\right\}=0 .
$$

Thus, by the same reasoning as in (2),

$$
H_{P}\left[T\left(B \cap B_{0}\right) \cap E\right] \leqq H_{P}\left[T\left(B \cap \varepsilon_{P}\right) \cap E\right] .
$$

(1) thus follows from (2) and (3).

2.5. Lemma. For $E \in \Gamma$ and any Borel set $B$ in the uv-plane,

$$
H^{2}\left[T\left(B \cap B_{0}\right) \cap E\right] \leqq \sum_{P \in U^{*}} H_{P}\left[T\left(B \cap \mathcal{E}_{P}\right) \cap E\right],
$$

where $U^{*}$ is the set of points $(1,0,0),(0,1,0),(0,0,1)$ on $U$ and $\varepsilon_{P}=\varepsilon_{P}(T)$.

Proof. By (iii) of $\$ 1.2$, for $P \in U, T\left(B \cap B_{0}\right) \cap E=A_{P}^{\prime} \cup A_{P}^{\prime \prime}$ where $A_{P}^{\prime}$, 
$A_{P}^{\prime \prime} \in \Gamma, A_{P}^{\prime} \cap A_{P}^{\prime \prime}=\varnothing, H_{P}\left[T\left(B \cap B_{0}\right) \cap E\right]=H_{P}\left(A_{P}^{\prime}\right)=H^{2}\left(A_{P}^{\prime}\right)$ and $H_{P}\left(A_{P}^{\prime \prime}\right)=0$. Then

(2) $T\left(B \cap B_{0}\right) \cap E=A \cup \underset{P \in U^{*}}{\cup} A_{P}^{\prime}, A=\bigcap_{P \in U^{*}} A_{P}^{\prime \prime}, L_{2} \pi_{P}(A)=0 \quad$ for $P \in U^{*}$.

Since $H^{2}(A) \leqq H^{2}\left(B_{0}\right)<\infty$, there is a Borel set $A^{*} \subset A$ such that $H^{2}\left(A^{*}\right)$ $=H^{2}(A)$. Set $B^{*}=T^{-1}\left(A^{*}\right) \cap B_{0}$. For $P \in U^{*}, N\left(x_{P}, T_{P}, B^{*}\right)=0$ for $x_{P} \notin \pi_{P}\left(A^{*}\right)$ and from (2), $L_{2} \pi_{P}\left(A^{*}\right) \leqq L_{2} \pi_{P}(A)=0$. Thus

$$
\begin{aligned}
H^{2}(A) & =H^{2}\left(A^{*}\right) \leqq \int N\left(x, T, B^{*}\right) d H^{2} \leqq \sum_{P \in U^{*}} \iint_{B^{*}}\left|J_{P}(u, v)\right| d u d v \\
& =\sum_{P \in U^{*}} \int N\left(x_{P}, T_{P}, B^{*}\right) d L_{2}=0 .
\end{aligned}
$$

Thus, from (2), (3) and the lemma in $\$ 2.4$,

$$
\begin{aligned}
H^{2}\left[T\left(B \cap B_{0}\right) \cap E\right] & \leqq \sum_{P \in U^{*}} H^{2}\left(A_{P}^{\prime}\right)=\sum_{P \in U^{*}} H_{P}\left[T\left(B \cap B_{0}\right) \cap E\right] \\
& =\sum_{P \in U^{*}} H_{P}\left[T\left(B \cap \varepsilon_{P}\right) \cap E\right],
\end{aligned}
$$

and (1) holds.

2.6. Lemma. An m.m.c. $\gamma$ under $T$ is an s.m.m.c. under $T$ if and only if for each set $O \in \Omega_{T}$ (see \$1.11) such that $\gamma \subset O$ we have $T(\gamma) \in D\left[T\left(O \cap B_{0}\right) \cdot\right]$.

Proof. Let $\gamma$ be an s.m.m.c. under $T$. Let $O \in \Omega_{T}$ be such that $\gamma \subset O$. Then $T(\gamma) \in D^{*}(T, O)$ and there is a positive integer $n_{0}$ such that for $m=1,2, \cdots$ there is a $P_{m} \in U$ such that for $\mathcal{E}_{P_{m}}=\mathcal{E}_{P_{m}}(T)$,

(1) $\quad H_{P_{m}}\left[T\left(O \cap \mathcal{E}_{P_{m}}\right) \cap s(T(\gamma), r)\right]>\pi r^{2} / n_{0} \quad$ for some $r, \quad 0<r<1 / m$.

By (i) of $\$ 1.2$ and the lemma in $\S 2.4$,

$$
\left.H^{2}\left[T\left(O \cap B_{0}\right) \cap s(T(\gamma), r)\right] \geqq H_{P_{m}} T\left(O \cap \mathcal{E}_{P_{m}}\right) \cap s(T(\gamma), r)\right] .
$$

From (2) and (1) it follows that $T(\gamma) \in D\left[T\left(O \cap B_{0}\right)\right]$.

Let $\gamma$ be an m.m.c. under $T$ such that for every $O \in \Omega_{T}$ such that $\gamma \subset O$, we have $T(\gamma) \in D\left[T\left(O \cap B_{0}\right)\right]$. By the lemma in $\S 2.5$,

(3) $H^{2}\left[T\left(O \cap B_{0}\right) \cap s(T(\gamma), r)\right] \leqq \sum_{P \in U^{*}} H_{P}\left[T\left(O \cap \varepsilon_{P}\right) \cap s(T(\gamma), r)\right] \quad$ for $r>0$.

Since $T(\gamma) \in D\left[T\left(O \cap B_{0}\right)\right]$ it follows from (3) that $T(\gamma) \in D_{P}\left[T\left(O \cap \varepsilon_{P}\right)\right]$ for some $P \in U^{*} \subset U$. By (ii) of the lemma in $\$ 1.9, T(\gamma) \in D^{*}[(T, O)]$. By the lemma in $\$ 1.12, \gamma$ is an s.m.m.c. under $T$.

2.7. Theorem. Let $T$ be a differentiable essentially absolutely continuous transformation from $Q$ into $S_{3}$ and let $S(T)$ be the union of the significant maxi- 
mal model continua under $T$. Then

$$
A(T)=\int N^{*}[x, T, \delta(T)] d H^{2} .
$$

Proof. By the lemma in $\$ 2.3$ and the set $B_{0}^{*}$ defined there we have that

$$
A(T)=\int N^{*}\left(x, T, B_{0}^{*}\right) d H^{2} .
$$

Then (1) will follow from (2) if for $S=S(T)$ we establish the equalities

$$
H^{2}\left[T\left(s-B_{0}^{*}\right)\right]=0 \quad \text { and } \quad H^{2}\left[T\left(B_{0}^{*}-\S\right)\right]=0 .
$$

For $E_{\infty}=\left[x \mid N^{*}\left(x, T, B_{0}^{*}\right)=\infty\right]$ we have

$$
H^{2}\left(E_{\infty}\right)=0 .
$$

Let $\gamma$ be an s.m.m.c. under $T$ such that $\gamma \subset\left(\delta-B_{0}^{*}\right), T(\gamma) \notin E_{\infty}$. Then there is an $O \in \Omega_{T}$ such that $\gamma \subset O, T(\gamma) \notin T\left(O \cap B_{0}\right)$. By the lemma in $\$ 2.6 T(\gamma)$ $\in\left\{D\left[T\left(O \cap B_{0}\right)\right]-T\left(O \cap B_{0}\right)\right\}$. Thus

$$
T\left(\mathcal{S}-B_{0}^{*}\right) \subset E_{\infty} \cup \underset{o \in \Omega_{T}}{\cup}\left\{D\left[T\left(O \cap B_{0}\right)\right]-T\left(O \cap B_{0}\right)\right\} .
$$

Since $H^{2}\left[T\left(B_{0}\right)\right]<\infty$, by (iv) of the lemma in $\S 1.5$ and by (4) the right side of (5) is the union of a denumerable number of sets of $H^{2}$ measure zero. Hence, the first equality of (3) is satisfied.

Let $\gamma$ be an m.m.c. under $T$ such that $\gamma \subset\left(B_{0}^{*}-\delta\right)$. By the lemma in $\S 2.6$ there is an $O \in \Omega_{T}$ such that $\gamma \subset O, T(\gamma) \in\left\{T\left(O \cap B_{0}\right)-D\left[T\left(O \cap B_{0}\right)\right]\right\}$. Thus

$$
T\left(B_{0}^{*}-s\right) \subset \underset{o \in \Omega_{T}}{\bigcup}\left\{T\left(O \cap B_{0}\right)-D\left[T\left(O \cap B_{0}\right)\right]\right\} .
$$

By (ii) of the lemma in $\S 1.5$ the right side of (6) is the union of a denumerable number of sets of $H^{2}$ measure zero. Hence the second equality of (3) is satisfied.

\section{FRÉCHET EQUIVALENT TRANSFORMATIONS}

3.1. Let $T$ and $T^{*}$ be two Fréchet equivalent continuous transformations from $Q$ into $S_{3}$ (see Rado [6, p. 57]). Then (see Youngs [10]) there exist simultaneous monotone-light factorizations of them

$$
T=l m, m: Q \Rightarrow M, l: M \rightarrow S_{3}, T^{*}=l m^{*}, m^{*}: Q \Rightarrow M, l: M \rightarrow S_{3},
$$

such that $m$ and $m^{*}$ are Fréchet equivalent. Let $a_{1}, a_{2}, \ldots$ be a dense denumerable set of points in $M$. For the open spheres $s\left(a_{i}, 1 / j\right)$ with center at $a_{i}$ and radius $1 / j$ we set 


$$
O_{i, j}=m^{-1}\left[s\left(a_{i}, 1 / j\right)\right], \quad O_{i, j}^{*}=m^{*-1}\left[s\left(a_{i}, 1 / j\right)\right], \quad i, j=1,2, \cdots .
$$

3.2. Lemma. Under the conditions of $\$ 3.1, \gamma$ is an m.m.c. under $T$ if and only if $m^{*-1} m(\gamma)$ is an m.m.c. under $T^{*}$ and $O_{i, j}^{*}=m^{*-1}\left[m\left(O_{i, j}\right)\right], O_{i, j}$ $=m^{-1} m^{*}\left(O_{i, j}^{*}\right), i, j=1,2, \cdots$.

Proof. The proof of the statements of the lemma follows from the definition of a monotone-light factorization.

3.3. Lemma. Under the conditions of $\$ 3.1$ for $P \in U, \mathcal{E}_{P}\left(T^{*}\right)$ $=m^{*-1}\left[m \varepsilon_{P}(T)\right]$.

Proof. See Mickle [5].

3.4. Lемма. Under the conditions of $\$ 3.1$ (see §1.8)

$$
D^{*}\left(T, O_{i, j}\right)=D^{*}\left(T^{*}, O_{i, j}^{*}\right), \quad i, j=1,2, \cdots .
$$

Proof. To prove (1) we need only to show that for $P \in U, \varepsilon_{P}=\varepsilon_{I^{\prime}}\left(T^{\prime}\right)$, $\mathcal{E}_{P}^{*}=\mathcal{E}_{P}\left(T^{*}\right)$

(2) $G_{s, t}\left[T\left(O_{i, j} \cap \varepsilon_{P}\right), P\right]=G_{s, t}\left[T\left(O_{i, j}^{*} \cap \varepsilon_{P}^{*}\right), P\right], \quad s, t, i, j=1,2, \cdots$

Since $O_{i, j}, \mathcal{E}_{P}$ are the union of m.m.c.'s under $T$ and $O_{i, j}^{*}, \varepsilon_{P}^{*}$ are the union of m.m.c.'s under $T^{*}, \quad m\left(O_{i, j} \cap \mathcal{E}_{P}\right)=m\left(O_{i, j}\right) \cap m\left(\mathcal{E}_{P}\right) \quad$ and $m^{*}\left(O_{i, j}^{*} \cap \mathcal{E}_{P}^{*}\right)$ $=m^{*}\left(O_{i, j}^{*}\right) \cap m^{*}\left(\mathcal{E}_{P}^{*}\right)$. Thus, by the lemmas in $\$ 3.2$ and $\S 3.3$

$$
\begin{aligned}
G_{s, t}\left[T\left(O_{i, j} \cap \mathcal{E}_{P}\right), P\right] & =G_{s, t}\left[\operatorname{lm}\left(O_{i, j} \cap \mathcal{E}_{P}\right), P\right]=G_{s, t}\left\{l\left[m\left(O_{i, j}\right) \cap m\left(\mathcal{E}_{P}\right)\right], P\right\} \\
& =G_{s, t}\left\{l\left[m^{*}\left(O_{i, j}^{*}\right) \cap m^{*}\left(\varepsilon_{P}^{*}\right)\right], P\right\} \\
& =G_{s, t}\left[\operatorname{lm}^{*}\left(O_{i, j}^{*} \cap \varepsilon_{P}^{*}\right), P\right]=G_{s, t}\left[T^{*}\left(O_{i, j}^{*} \cap \varepsilon_{P}^{*}\right), P\right]
\end{aligned}
$$

for $s, t, i, j=1,2, \cdots$, and (2) holds.

3.5. LEMMA. Under the.conditions of $\$ 3.1$ an m.m.c. $\gamma$ under $T$ is an s.m.m.c. under $T$ if and only if $\gamma^{*}=m^{*-1} m(\gamma)$ is an s.m.m.c. under $T^{*}$.

Proof. Let $\gamma$ be an s.m.m.c. under $T$. Let $O_{i, j}^{*}$ be such that $\gamma^{*}=m^{*-1} m(\gamma)$ $\subset O_{i, j}^{*}$. By the lemmas in $\$ 3.2$ and $\$ 3.4, \gamma \subset O_{i, j}, T^{*}\left(\gamma^{*}\right)=T(\gamma) \in D^{*}\left(T, O_{i, j}\right)$ $=D^{*}\left(T^{*}, O_{i, j}^{*}\right)$. By the lemma in $\S 1.12, \gamma^{*}$ is an s.m.m.c. under $T^{*}$.

Let $\gamma$ be an m.m.c. under $T$. If $\gamma^{*}=m^{*-1} m(\gamma)$ is an s.m.m.c. under $T^{*}$ then the fact that $\gamma$ is an s.m.m.c. under $T$ follows by the same argument.

3.6. ThEOREM. Let $T$ and $T^{*}$ be two Fréchet equivalent continuous transformations from $Q$ into $S_{3}$. Then

$$
N^{*}[x, T, s(T)]=N^{*}\left[x, T^{*}, s\left(T^{*}\right)\right] \quad \text { for } x \in S_{3} .
$$

Proof. The proof of the statement of the theorem follows directly from the lemma in $\S 3.5$. 
3.7. THEOREM. Let $T$ be a continuous transformation from the unit square $Q: 0 \leqq u \leqq 1,0 \leqq v \leqq 1$ in the uv-plane into Euclidean three space $S_{3}$. The Lebesgue area $A(T)$ of the surface represented by $T$ is given by the formula

$$
A(T)=\int N^{*}[x, T, \delta(T)] d H^{2},
$$

where $S(T)$ is the union of the significant maximal continua under $T$ as defined in $\$ 1.10$.

Proof. Case 1. $A(T)=\infty$. (1) then follows from the theorem in $\S 1.15$.

Case 2. $A(T)<\infty$. By Cesari [2] there is a differentiable essentially absolutely continuous transformation $T^{*}$ from $Q$ into $S_{3}$ that is Fréchet equivalent to $T$. Then $A(T)=A\left(T^{*}\right)$ (see Rado [6, p. 468]) and by the theorems in $\S 3.6$ and $\S 2.7$

$$
A(T)=A\left(T^{*}\right)=\int N^{*}\left[x, T^{*}, \mathrm{~s}\left(T^{*}\right)\right] d H^{2}=\int N^{*}[x, T, \mathrm{~s}(T)] d H^{2} .
$$

Thus (1) holds.

\section{BIBLIOGRAPHY}

1. L. Cesari, Caratterizzazione analitica delle superficie continue di area finita secondo Lebesgue, Annali della R. Scuola Normale Superiore di Pisa (2) vol. 10 (1941) pp. 253-294 and vol. 11 (1942) pp. 1-42.

2. - On the representation of surfaces, Amer. J. Math. vol. 72 (1950) pp. 335-346.

3. H. Federer, Surface area. II, Trans. Amer. Math. Soc. vol. 55 (1944) pp. 438-456.

4. - Measure and area, Bull. Amer. Math. Soc. vol. 58 (1952) pp. 306-378.

5. E. J. Mickle, Lebesgue area and Hausdorf measure, Rend. Circ. Mat. Palermo, Series II, Tomo IV (1955) pp. 205-236.

6. T. Rado, Length and area, Amer. Math. Soc. Colloquim Publications, vol. 30, 1948.

7. Two-dimensional concepts of bounded variation and absolute continuity, Duke Math. J. vol. 14 (1949) pp. 587-608.

8. - On essentially absolutely continuous plane transformations, Bull. Amer. Math. Soc. vol. 55 (1949) pp. 629-632.

9. - On multiplicity functions associated with Lebesgue area, Rend. Circ. Mat. Palermo, Series II, Tomo IV (1955) pp. 219-236.

10. J. W. T. Youngs, A reduction theorem concerning the representation problem for Fréchet varieties, Proc. Nat. Acad. Sci. U.S.A. vol. 32 (1946) pp. 328-330.

The Ohio State University, Columbus, Оhiо 